\title{
Construction Safety Evaluation for Prefabricated Concrete-Constructions based on Attribute Mathematics
}

\author{
CHANG Chunguang $^{1, a}$, YANG Shuang ${ }^{1, b}$, and LUO Jingyu ${ }^{2, c}$ \\ ${ }^{1}$ School of Management, Shenyang Jianzhu University, Shenyang 110168, China \\ ${ }^{2}$ School of Information and Control Engineering, Shenyang Jianzhu University, Shenyang 110168 , \\ China \\ achang_chunguang@163.com, bccg777@sohu.com, cccg7788@sohu.com
}

Keywords: prefabricated building; attribute mathematics; safety evaluation

\begin{abstract}
This paper applies the attribute mathematics theory to prefabricated building construction safety evaluation, establishes the attribute mathematics model of prefabricated building construction safety evaluation. Based on the analysis of main causes of prefabricated building construction safety, personnel quality, technology and equipment, safety management, environment are chosen as the Level I indexes, and four Level I indexes divide into several Level II indexes.Calculating the single indexes attribute measure by establishing attribute measure functions. To calculate the comprehensive attribute measure, the weight of the evaluation index is determined by using the analytic hierarchy process (AHP). Finally, the safety of the target project ares recognized by the confidence criterion.Take a concrete project as an example to explain how to use the model to evaluate prefabricated building construction safety.
\end{abstract}

\section{Introduction}

The prefabricated building is a building constructed by assembling, jointing the prefabricated parts in the factory and partially casting in the with the traditional building,the prefabricated building saves energy and resources, makes the construction process more environmentally friendly, improves the technology content of the construction industry, is in accordance with the development strategy of green architecture and architectural indusrtialiaztion, and meets the requirements of sustainable development of construction industry. The prefabricated building is in the developing stage, and the construction safety management must be solved to develop prefabricated building. Establishing prefabricated building construction safety evaluation system is only one part of the safety management work, only by establishing scientific and accurate prefabricated building construction safety evaluation system to make accurate judgment of safety degree of prefabricated building construction. However, current research on prefabricated building construction safety evaluation is still at the initial stage,there are problems such as the evaluation indexes system is not comprehensive enough ,evaluation method is not scientific,etc. Therefore, it is necessary to study prefabricated building construction safety evaluation, propose a scientific and accurate evaluation system and evaluation methods.

Although the study of prefabricated building construction safety is less, but some achievements were made in the traditional building evaluation, and proposed a series of evaluation methods,such as analytic hierarchy process (AHP)[1], gualitative risk analysis[2], grey relation model[3], task demands assessment(TDA)[4]. But the large and small operations of the fuzzy sets in the fuzzy comprehensive evaluation result in the loss of some intermediate information.Lead to the phenomenon of unclear level division and evaluation of the structure is irrational[5]. This paper establishes the safety evaluation indexes system for prefabricated building construction, determines the weights of indexes by using the analytic hierarchy process,evaluates the safety of the prefabricated building construction by using attribute mathematics evaluation method. 


\section{Establish prefabricated building construction safety evaluation}

Establish evaluation indexes system. According to the construction features of prefabricated building and the regulations of prefabricated building safety established in various provinces and cities,by surveying and analysing the prefabricated building safety situation,the safety evaluation system as shown in table 1.

Table 1 Prefabricated building safety evaluation indexes system

\begin{tabular}{|c|c|}
\hline Level Iindexes & Level IIindexes \\
\hline \multirow{4}{*}{ Personnel quality } & Quality level of employee culture \\
\hline & Technical level of employees \\
\hline & Safety education and training situation \\
\hline & Enterprise qualification and qualification of employees \\
\hline \multirow{5}{*}{$\begin{array}{c}\text { Technology } \\
\text { and equipment }\end{array}$} & Large equipment installation and safety control \\
\hline & Maintenance of construction equipment \\
\hline & Safety protection facilities \\
\hline & Security sign setting \\
\hline & Construction technology maturity degree \\
\hline \multirow{5}{*}{ Safety management } & Safety production rules and regulations \\
\hline & Organization for safety production management \\
\hline & Safety supervision and inspection \\
\hline & Risk source and component stacking management \\
\hline & Accident prevention and emergency management \\
\hline \multirow{3}{*}{ Environment } & The natural environment \\
\hline & The working environment \\
\hline & The surrounding environment \\
\hline
\end{tabular}

The division of evaluation level. The safety of prefabricated building is divided into 5 evaluation levels:Excellent $C_{1}$, Good $C_{2}$, General $C_{3}$,Poor $C_{4}$, Failure $C_{5}$. The 5 evaluation levels constitute the level set $\mathrm{U}=\left(C_{1}, C_{2}, C_{3}, C_{4}, C_{5}\right)$, the standard of each indexes of evaluation scores of concrete as shown in table 2.The specific standard to classify the level of indexes are shown in Table 2.

Table 2 Evaluation standard and classification standard

\begin{tabular}{|c|c|c|c|c|}
\hline \multicolumn{5}{|c|}{ Evaluation Level } \\
\hline Excellent & Good & General & Poor & Failure \\
\hline $100-90$ & $90-80$ & $80-70$ & $70-60$ & $60-0$ \\
\hline
\end{tabular}

\section{Prefabricated building construction safety evaluation method}

References Let $X$ be a collection of objects to be evaluated. Each element in $X$ has the characteristic $m$ indexes $\left(I_{1}, I_{2}, \ldots, I_{m}\right)$. The elements in $\mathrm{X} \operatorname{set}\left(C_{1}, C_{2}, \ldots, C_{K}\right)$. Cn as the evaluation grade $^{[7]}$.The extent of the evaluation object to level k expressed by attribute measure ${ }^{\mu_{k}}$. $\mu_{j k}$ denotes the extent belonging to level $k$ of index $j$ by attribute measure.

Single index attribute measure. We use the number $\mu(x \in A)$ or $\mu(A)$ to quantitatively describe the extent of the "x having attribute $A$ ".Because of convenience, the attribute measure is 
between [0,1].Different attribute sets can give the corresponding attribute measure.But the attribute measure must subject to

$$
\begin{aligned}
& \mu_{k} \geq 0, \sum_{\mathrm{k}=1}^{K} \mu_{k}=1 \\
& \mu_{j \mathrm{k}} \geq 0, \sum_{\mathrm{k}=1}^{K} \mu_{\mathrm{j} k}=1
\end{aligned}
$$

The evaluation grades for each index are usually represented by a number.The evaluation standards are expressed in the form of table 3, which is actually a single indexes grade partition .

\begin{tabular}{|c|c|c|c|c|}
\hline Level Index & $\mathrm{C}_{1}$ & $\mathrm{C}_{2}$ & $\ldots$ & $\mathrm{C}_{\mathrm{K}}$ \\
\hline$\overline{I_{1}}$ & $\mathrm{a}_{10}-\mathrm{a}_{11}$ & $\mathrm{a}_{11}-\mathrm{a}_{12}$ & $\ldots$ & $\mathrm{a}_{1 \mathrm{~K}-1}-\mathrm{a}_{1 \mathrm{~K}}$ \\
\hline $\mathrm{I}_{2}$ & $\mathrm{a}_{20}-\mathrm{a}_{21}$ & $\mathrm{a}_{21}-\mathrm{a}_{22}$ & $\ldots$ & $\mathrm{a}_{2 \mathrm{~K}-1}-\mathrm{a}_{2 \mathrm{~K}}$ \\
\hline \multicolumn{5}{|c|}{$\ldots \ldots$} \\
\hline $\mathrm{I}_{\mathrm{m}}$ & $\mathrm{a}_{\mathrm{m} 0}-\mathrm{a}_{\mathrm{m} 1}$ & $\mathrm{a}_{\mathrm{m} 1}-\mathrm{a}_{\mathrm{m} 2}$ & $\ldots$ & $a_{m K-1}-a_{m K}$ \\
\hline
\end{tabular}

Table 3 Single index classification table

Single indexes attribute measure function $\mu_{\mathrm{jk}}(\mathrm{t})$ was established by table 3.In table $3, a_{j k}$ subjects to $a_{j 0}<a_{j 1}<\cdots<a_{j K}$ or $a_{j 0}>a_{j 1}>\cdots>a_{j K}$.

$$
\begin{aligned}
& b_{j k}=\frac{a_{j k-1}+a_{j k}}{2}, k=1,2, \ldots, K \\
& d_{j k}=\min \left(\left|b_{j k}-a_{j k}\right|,\left|b_{j k+1}-a_{j k}\right|\right), k=1,2, \cdots, K-1
\end{aligned}
$$

The single evaluation indexes attribute measure function is as follows:

$$
\begin{aligned}
& \mu_{j 1}(t)=\left\{\begin{array}{cc}
1, & t<a_{j 1}-d_{j 1} \\
\frac{\left|t-a_{j 1}-d_{j 1}\right|}{2 d_{j 1}}, & a_{j 1}-d_{j 1} \leq t \leq a_{j 1}+d_{j 1} \\
0, & a_{j 1}-d_{j 1}<t
\end{array}\right. \\
& \mu_{j K}(t)=\left\{\begin{array}{cl}
1, & a_{j K-1}+d_{j K-1}<t \\
\frac{\left|t-a_{j K-1}+d_{j K-1}\right|}{2 d_{j K-1}}, & a_{j K-1}-d_{j K-1} \leq t \leq a_{j K-1}+d_{j K-1} \\
0, & t<a_{j K-1}-d_{j K-1}
\end{array}\right. \\
& \mu_{j k}(t)=\left\{\begin{array}{cc}
0, & t<a_{j i-1}-d_{j i-1} \\
\frac{\left|t-a_{j k-1}+d_{j k-1}\right|}{2 d_{j k-1}}, & a_{j k-1}-d_{j k-1} \leq t \leq a_{j k-1}+d_{j k-1} \\
1, & a_{j k-1}+d_{j k-1}<t<a_{j k}-d_{j k} \\
\frac{\left|t-a_{j k}+d_{j k}\right|}{2 d_{j k}}, & a_{j k}-d_{j k} \leq t \leq a_{j k}+d_{j k} \\
0, & a_{j k}+d_{j k}<t
\end{array}\right.
\end{aligned}
$$

Where, $j=1,2, \cdots, m ; k=2,3, \cdots K-1$. From the above formula can be known any $t, \mu_{j k}(t)$ subjects to formula1.

Multi-indexes attribute measure. If it is known each single indexes $I_{j}(1 \leq i \leq m)$ of sample $x$ and each single indexes attribute measure $\mu_{j k}=\mu \quad\left(x_{j} \in C_{k}\right)$ of sample $x$.The weight of the $j$ indexes is $\omega_{j}, \omega_{j}$ comply with:

$$
\omega_{\mathrm{j}} \geq 0, \sum_{j=1}^{m} \omega_{j}=1
$$

By weighted sum, multi-indexes attribute $\mu_{k}=\sum_{j=i}^{m} \omega_{j} \mu_{j k}$ can be obtained by indexes weights $\omega_{j}$ and single index attribute measure $\mu_{x j k}$. 
Attribute recognition analysis. After the attribute measure $\mu_{k}=\mu \quad\left(x \in C_{k}\right), 1 \leq k \leq K$ of sample $x$ is obtained, two common recognition standards are used to determine the level of the $X$, and the two standards are the minimum price criterion and the confidence criterion.For orderly evaluation set $\left(C_{1}, C_{2}, \cdots, C_{K}\right)$ adopt confidence criterion to identify the level of sample $x$.In prefabricated building construction safety evaluation, the evaluation set $\left(C_{1}, C_{2}, \cdots, C_{K}\right)$ is set as an ordered set.For example, in prefabricated building construction safety evaluation,the evaluation set is $C_{1}=\{$ very safe $\}, C_{2}=\{$ safe $\}, C_{3}=$ \{unsafe $\}$. Generally consider "very safe" better or stronger than "safe" ,record as $C_{1}>C_{2}$.In the same way, $C_{2}>C_{3}$.If set $C_{1}=\{$ unsafe $\}, C_{2}=\{$ safe $\}, C_{3}=\{$ very safe $\}$, then $C_{1}<C_{2}<C_{3}$. Set the evaluation set for the ordered set,assume $C_{1}>C_{2}>, \cdots,>C_{\mathrm{x}}$, confidence is $\lambda, 0.5<\lambda \leq 1$.If $k_{0}=\min \left\{k \mid \sum_{i=1}^{k} \mu_{i l} \geq \lambda, 1 \leq k \leq K\right\}$, it is considered that $\mu$ belongs to level $C_{k 0}$.

The above standard require "strong" class or level of a considerable proportion.In application, the confidence level $\lambda$ generally takes between 0.6 and 0.7 .

\section{Application Example}

Single indexes attribute measure function. In application, according to the relationship between the safety indexes of the construction safety indexes, the construction safety evaluation standard of the prefabricated building construction is shown in Table 1.According to the above formula, single indexes attribute measure functions are as follows:

$$
\begin{aligned}
& \mu_{11}(t)=\left\{\begin{array}{ccc}
1 & 95<t \\
\frac{t-85}{10} & 85 \leq t \leq 95 \\
0 & t<85
\end{array} ; \mu_{12}(t)=\left\{\begin{array}{cc}
0 & 95<t \\
\frac{95-\mathrm{t}}{10} & 85 \leq t \leq 95 \\
\frac{\mathrm{t}-75}{10} & 75 \leq t \leq 85 \\
0 & t<75
\end{array} ; \mu_{13}(t)=\left\{\begin{array}{cc}
0 & 85<t \\
\frac{85-\mathrm{t}}{10} & 75 \leq t \leq 85 \\
\frac{\mathrm{t}-65}{10} & 65 \leq t \leq 75 \\
0 & t<75
\end{array} ;\right.\right.\right. \\
& \mu_{14}(t)=\left\{\begin{array}{cc}
0 & 75<t \\
\frac{75-\mathrm{t}}{10} & 65 \leq t \leq 75 \\
\frac{\mathrm{t}-55}{10} & 55 \leq t \leq 65 \\
0 & t<55
\end{array} ; \mu_{15}(t)=\left\{\begin{array}{cc}
0 & 65<t \\
\frac{65-t}{10} & 55 \leq t \leq 65 \\
1 & t<55
\end{array}\right.\right.
\end{aligned}
$$

The single indexes attribute measure functions of $I I_{2} \sim I I_{17}$ are the same as $I I_{1}$.

Weights of indexes. In this paper, the weights of II level indexes correspond to the I level indexes are determined by using analytic hierarchy process, as shown in Table 4. 
Table 4 Safety evaluation indexes weights

\begin{tabular}{|c|c|c|c|}
\hline Level I indexes & Weights & Level II indexes & Weights \\
\hline \multirow{4}{*}{ Personnel quality } & \multirow{4}{*}{0.242} & Quality level of employee culture & 0.213 \\
\hline & & Technical level of employees & 0.276 \\
\hline & & Safety education and training situation & 0.258 \\
\hline & & Enterprise qualification and qualification of employees & 0.253 \\
\hline \multirow{5}{*}{$\begin{array}{c}\text { Technology } \\
\text { and equipment }\end{array}$} & \multirow{5}{*}{0.261} & Large equipment installation and safety control & 0.216 \\
\hline & & Maintenance of construction equipment & 0.225 \\
\hline & & Safety protection facilities & 0.213 \\
\hline & & Security sign setting & 0.178 \\
\hline & & Construction technology maturity degree & 0.168 \\
\hline \multirow{5}{*}{ Safety management } & \multirow{5}{*}{0.266} & Safety production rules and regulations & 0.187 \\
\hline & & Organization for safety production management & 0.179 \\
\hline & & Safety supervision and inspection & 0.216 \\
\hline & & Risk source and component stacking management & 0.207 \\
\hline & & Accident prevention and emergency management & 0.211 \\
\hline \multirow{3}{*}{ Environment } & \multirow{3}{*}{0.231} & The natural environment & 0.36 \\
\hline & & The working environment & 0.34 \\
\hline & & The surrounding environment & 0.3 \\
\hline
\end{tabular}

Case Study. Comprehensive evaluation of prefabricated building construction safety projects.Randomly selected 40 experts and engineers to fill in project safety questionnaire.The score of each evaluation indexes as shown in table 5 after statistical analysis software processing data. 
Table 5 Weights and scores of indexes

\begin{tabular}{|c|c|c|}
\hline Level II indexes & Weights of Level II indexes & scores \\
\hline Quality level of employee culture & 0.213 & 87 \\
\hline Technical level of employees & 0.276 & 82 \\
\hline Safety education and training situation & 0.258 & 79 \\
\hline Enterprise qualification and qualification of employees & 0.253 & 83 \\
\hline Large equipment installation and safety control & 0.216 & 89 \\
\hline Maintenance of construction equipment & 0.225 & 91 \\
\hline Safety protection facilities & 0.213 & 85 \\
\hline Security sign setting & 0.178 & 79 \\
\hline Construction technology maturity degree & 0.168 & 84 \\
\hline Safety production rules and regulations & 0.187 & 81 \\
\hline Organization for safety production management & 0.179 & 85 \\
\hline Safety supervision and inspection & 0.216 & 87 \\
\hline Risk source and component stacking management & 0.207 & 89 \\
\hline Accident prevention and emergency management & 0.211 & 81 \\
\hline The natural environment & 0.36 & 87 \\
\hline The working environment & 0.34 & 82 \\
\hline The surrounding environment & 0.3 & 84 \\
\hline
\end{tabular}

The attribute measure of prefabricated building construction safety evaluation index is calculated according to the measure function of individual index attribute. The results are $I I_{1}=(0.2,0.8,0,0,0)$ $I I_{2}=(0,0.7,0.3,0,0) ; I I_{3}=(0,0.4,0.6,0,0) ; I I_{4}=(0,0.8,0.2,0,0) ; I I_{5}=(0.4,0.6,0,0,0) ; \quad I I_{6}=(0.6,0.4,0,0,0)$ $I I_{7}=(0,1,0,0,0) ; \quad I I_{8}=(0,0.4,0.6,0,0) ; \quad I I_{9}=(0,0.9,0.1,0,0) ; I I_{10}=(0,0.6,0.4,0,0) ; I I_{11}=(0,1,0,0,0)$ $I I_{12}=(0.2,0.8,0,0,0) \quad ; \quad I I_{13}=(0.4,0.6,0,0,0) ; \quad I I_{14}=(0,0.6,0.4,0,0) \quad ; \quad I I_{15}=(0.2,0.8,0,0,0)$; $I I_{16}=(0,0.7,0.3,0,0) ; \quad I I_{17}=(0,0.9,0.1,0,0)$. Then calculate the comprehensive attribute measure $\left(\mu_{x 1}, \mu_{\times 2}, \mu_{\times 3}, \mu_{\times 4}\right)=(0.118242,0.70692,0.17484,0,0)$. According to comprehensive attribute measure, the reliability of the project belongs to excellent is 0.118242 .The reliability of belongs to good is $0.118242+0.70692=0.82516$.Even taking the high confidence level of 0.8 , the project safe level is still good.Generally the confidence to take between 0.6 and 0.7 , the result of the prefabricated building construction safety evaluation is determined as good.

\section{Conclusion}

In Based on the theory of attribute mathematics, the model of prefabricated building construction safety evaluation is established. Providing an idea of prefabricated building construction safety evaluation.Quantitative methods to evaluate prefabricated building construction safety, making the evaluation results more accurate and reliable.Prefabricated building construction safety is evaluated by using the attribute mathematical model established in this paper.Can be seen from the calculation results, the method of attribute mathematics can be used to evaluate prefabricated building construction safety. 


\section{Acknowledgements}

This work was financially supported by the Project from Ministry of Housing and Urban-Rural Development of the People's Republic of China(2014-R3-014), Science Research Program of Liaoning Province Education Administration: Liaoning city public safety management by E-CBR (W2014084) \& Research on the industry-university-research-user cooperative innovation mechanism of the market oriented research in Liaoning (W2014085), Work Safety Project of State Administration (2013-13), Shenyang Scientific and Technological Planning (F13-317-5-12; F14-230-5-19; F15-198-5-15)and Science Research Foundation of Shenyang Jianzhu University (2014035).

\section{References}

[1] Hinze Jimmie,Thurman Samuel,Wehle Andrew: Leading indicators of contruction safety performance. Safety Science, Vol. 51 (2013), p. 23-28

[2] Nima Gerami, Heravi Gholamreza: Safe floats identification using qualitative risk analysis in construction projects schedulingSeresht. Proceedings, Annual Conference - Canadian Society for Civil Engineering, Vol. 3(2011), p 2080-2089

[3] Zhou Ru, Zhang Wei: Model of improved grey relation of safety evaluation in high-rise building fires. International Review on Computers and Software, Vol. 7(2012), p. 2634-2638

[4] Lee Hyun-Soo, Kim Hyunsoo, Park Moonseo, Lee Kwang-Pyo: Construction risk assessment using site influence factors. Journal of Computing in Civil Engineering, Vol. 26(2012), p. 319-330

[5] Cai Min, Chen Xingying, Liu Jian, et al.: Operation state evaluation of distribution network based on attribute mathematics. Applied Mechanics and Materials, Vol. 672-674(2014), p. 1037-1041 